\title{
The frequencies and disparities of adverse childhood experiences in the U.S.
}

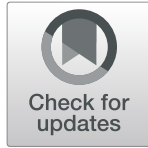

\author{
Zachary Giano* (D), Denna L. Wheeler and Randolph D. Hubach
}

\begin{abstract}
Background: Adversity experienced during childhood manifests deleteriously across the lifespan. This study provides updated frequency estimates of ACEs using the most comprehensive and geographically diverse sample to date.

Methods: ACEs data were collected via BRFSS (Behavioral Risk Factor Surveillance System). Data from a total of 211, 376 adults across 34 states were analyzed. The ACEs survey is comprised of 8 domains: physical/emotional/sexual abuse, household mental illness, household substance use, household domestic violence, incarcerated household member, and parental separation/divorce. Frequencies were calculated for each domain and summed to derive mean ACE scores. Findings were weighted and stratified by demographic variables. Group differences were assessed by post-estimation F-tests.

Results: Most individuals experienced at least one ACE (57.8\%) with 21.5\% experiencing 3+ ACEs. F-tests showed females had significantly higher ACEs than males (1.64 to 1.46). Multiracial individuals had a significantly higher ACEs (2.39) than all other races/ethnicities, while White individuals had significantly lower mean ACE scores (1.53) than Black (1.66) or Hispanic (1.63) individuals. The 25-to-34 age group had a significantly higher mean ACE score than any other group (1.98). Generally, those with higher income/educational attainment had lower mean ACE scores than those with lower income/educational attainment. Sexual minority individuals had higher ACEs than straight individuals, with significantly higher ACEs in bisexual individuals (3.01).
\end{abstract}

Conclusion: Findings highlight that childhood adversity is common across sociodemographic, yet higher in certain categories. Identifying at-risk populations for higher ACEs is essential to improving the health outcomes and attainment across the lifespan.

Keywords: Adverse childhood experiences, BRFSS

\section{Background}

Mental and physical health, disease, cognition, wellbeing, and lifelong health is rooted in childhood. The study of adverse childhood experiences (ACEs) and the panoply of risks associated with these adverse events has grown markedly in the past 20 years [1]. The study of individuals with high ACEs has revealed significant physical health risks such as heart and pulmonary diseases,

\footnotetext{
* Correspondence: Giano@okstate.edu

Center for Rural Health, Oklahoma State University--Center for Health Sciences, 1111 W 17th Street, Tulsa, OK 74107, USA
}

lung cancer, metabolic issues, inflammation, and liver diseases [2-6]. Mental health is equally affected by ACEs, as studies show strong links to depression, anxiety, severe mood disorders, and suicide [7-10]. Nevertheless, demographic diversity within these studies have been limited, with researchers calling for updated prevalence rates regarding ACEs by demographics and region [11].

Considerable research has been devoted to adverse childhood experiences because of its strong associations to public health issues. Within a public health context, ACEs have been linked to homelessness [12], lifetime

\section{$\triangle B M C$}

(c) The Author(s). 2020 Open Access This article is licensed under a Creative Commons Attribution 4.0 International License, which permits use, sharing, adaptation, distribution and reproduction in any medium or format, as long as you give appropriate credit to the original author(s) and the source, provide a link to the Creative Commons licence, and indicate if changes were made. The images or other third party material in this article are included in the article's Creative Commons licence, unless indicated otherwise in a credit line to the material. If material is not included in the article's Creative Commons licence and your intended use is not permitted by statutory regulation or exceeds the permitted use, you will need to obtain permission directly from the copyright holder. To view a copy of this licence, visit http://creativecommons.org/licenses/by/4.0/ The Creative Commons Public Domain Dedication waiver (http://creativecommons.org/publicdomain/zero/1.0/) applies to the data made available in this article, unless otherwise stated in a credit line to the data. 
alcohol dependence [13], opioid addiction [14], and increased exposure to HIV risk $[15,16]$. Though these investigations have been a critical step in the development of health programming to attenuate these outcomes, more recent research regarding ACEs in the context of public health have revealed that while it is generally accepted that ACEs have a cumulative effect, not all populations are equally vulnerable to ACEs [11], and further, certain segments of the population may manifest childhood adversity differently [17] (e.g., one study found that the adverse mental health impact of ACEs on Whites was consistently greater than on Black and Latino individuals), thus suggesting a more complex relationship than traditional linear relationships with ACEs show in the general population. As such, ACEs prevention programming with a public health emphasis has shifted to more tailored-specific programming for specific races/ ethnicities and has shown promising results in Black and Hispanic communities [18, 19].

Starting in 2009, the Centers for Disease Control (CDC) gave states the option to collect ACEs data as a part of the Behavioral Risk Factor Surveillance Survey (BRFSS), a national survey of demographics, behaviors, and health indicators [20]. As a product of these surveys, Merrick and colleagues [11] collected the most comprehensive ACEs data to date, acquiring ACEs data from over 200,000 individuals in 23 states from the years 2011 to 2014. Using the same methodology, we have collected ACEs data in the same way.

Compared to Merrick's and colleagues' study, our study is methodologically expanded in four important ways that help broaden the depth of ACEs prevalence. The first is that we collected data from 11 additional states that were not included in Merrick et al's study (a $48 \%$ increase). With the additions of New Mexico, West Virginia, Kentucky, Ohio, Texas, Arkansas, Georgia, Hawaii, Louisiana, New York, and Illinois, we believe this expands the breadth of geographic available data on the topic and to our knowledge, is the most current and geographically comprehensive ACEs database to date. In particular, states classified in the South are especially understudied with respect to ACEs. For example, although Merrick and colleagues' article was the most geographic expansive article to date, their analysis only included 5 of the 16 states in the South (31\%), while our investigation includes 12 of the 16 states (75\%). This is important due to preliminary data suggesting that southern states may have higher rates of adversity among children compared to other regions [21].

Second, among states already represented in both Merrick's study and our study, we collected updated data from 13 states (Alaska, Arizona, California, Connecticut, Iowa, Michigan, Nevada, Oklahoma, Oregon, Pennsylvania, South Carolina, Tennessee, and Wisconsin). Third, our study limited data to a single year. Merrick et al. used several years of data for a single state (this was the case for eight states in their study), whereas ours only allows for the latest year of each state counting only once. We believe that using multiple years of the same state possibly inflates the data from that region and also may account for duplicative data (e.g., people may have taken the survey twice and thus were counted twice in their analyses, overrepresentation of a particular state and/or region, unbalanced racial/ethnic categories, etc.). Lastly, Merrick's study did not utilize any type of significance testing. We further expand on their methods by utilizing post-estimation F-tests to assess differences in ACEs prevalence among demographic variables in order to detect significant differences among groups.

Conceptually, we view the demographic characteristics of individuals bifurcated into two levels which interface with ACEs (see Fig. 1). First, static demographic characteristics are elements which are generally inherent to individuals. These characteristics include gender, race/ ethnicity, sexual orientation, geographic residence, and birth year. Next, dynamic demographic differences are traits which change in a more active way beyond childhood, particularly after ACEs. While ACEs have been linked to lifelong outcomes beyond both demographic categories, $[1,22]$ there may be statistical differences in ACEs by static demographic characteristics, while dynamic demographic characteristics may be influenced by other dynamic demographic characteristics (e.g., education influencing future income), static demographic characteristics (e.g., the effects of gender discrimination on income), and ACEs (e.g., childhood adversity affecting future income) [23, 24]. Moreover, understanding the impact that ACEs has on lifelong outcomes can be better understood by the stratification of individual elements from both categories among ACEs. This is particularly true for the development of prevention/ intervention programs centered on ACEs, as programs which are tailored by demographic characteristics have shown greater efficacy [25].

\section{Methods}

Data were obtained from the CDC's BRFSS; a yearly, national survey that collects data via cellular and landline telephone interviews among adults 18 years and older. The BRFSS uses a multistage sampling design to acquire data on health-related domains from noninstitutionalized civilian populations residing in all 50 states, the District of Columbia, and three U.S. territories (Puerto Rico, Guam, and the U.S. Virgin Islands). The BRFSS has three overall modules: 1 . core modules are sets of survey questions consistently administered to all states and territories, 2. optional modules which consist of $\mathrm{CDC}$ developed questions that states can include in 


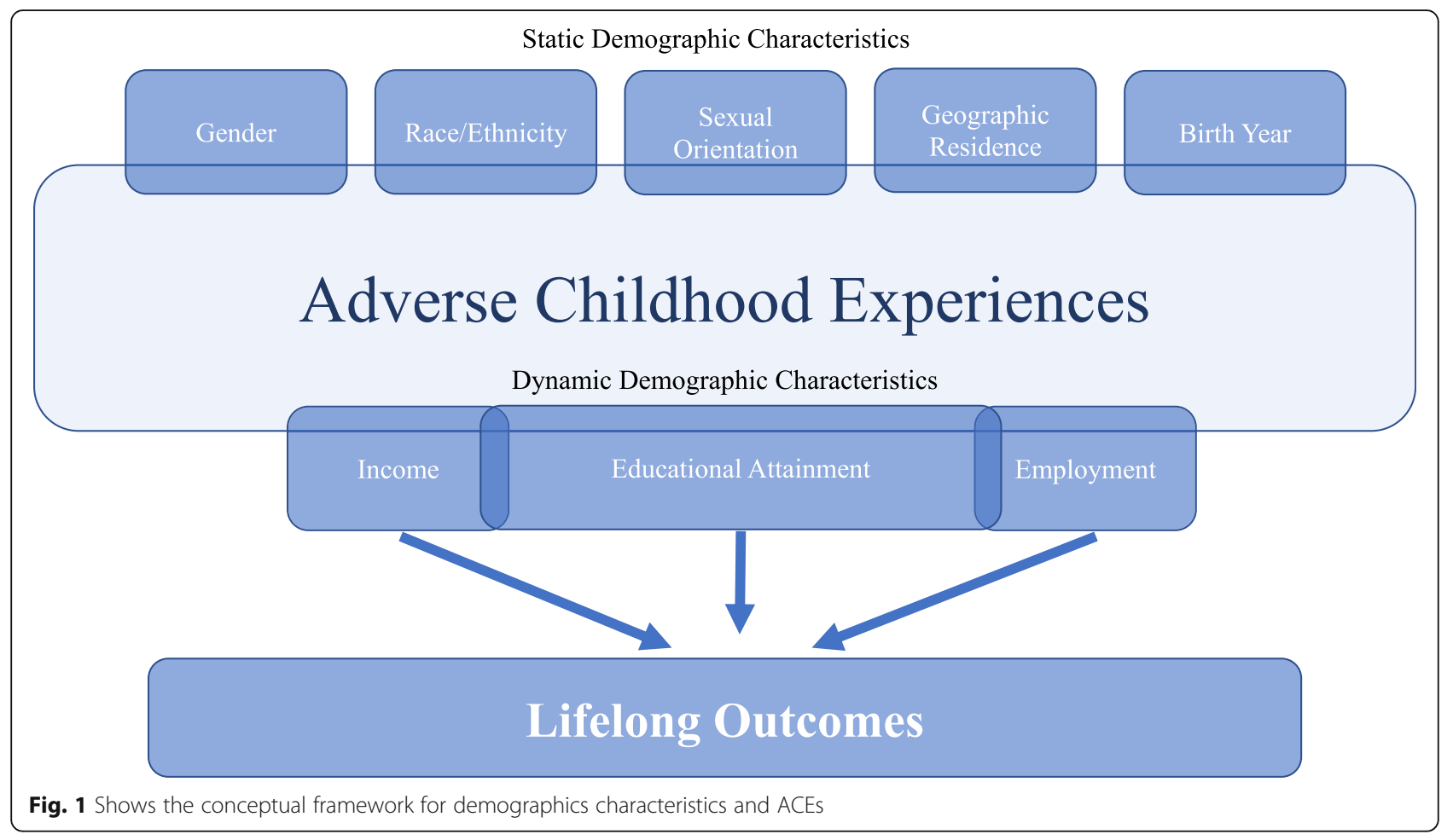

their survey, and 3. state-added questions, which include customizable items developed by each state coordinator. Only the core modules are publicly available. ACEs data was collected as a part of the optional modules. As of 2018, 41 states collected ACE data. It should be noted that several states have collected 2018 data for the first time, however, this data is typically not available until at least 2 years after collection.

The ACEs module consists of 11 questions derived from the CDC's ACEs study investigating adverse events in childhood before the age of 18 [26]. The survey questions fall into eight adversity domains including emotional abuse, physical abuse, sexual abuse, intimate partner violence (IPV), household substance use, household mental illness, parental separation/divorce, and household members who are incarcerated. The responses were dichotomized and summed, thus creating an ACE score range of 0 to 8 (higher scores indicating greater exposure to adverse events). Ford and colleagues offer an in-depth description of the BRFSS ACE module, factorial structure, and calculated ACE scores [27].

In total, 38 out of 50 states collected ACEs data starting from 2011 to 2017 (Washington D.C. did not collect data). Of these, three states declined to share data for various reasons (e.g., stopped giving data due to lack of resources, data is privately funded and not given publicly, committee declined to have data included in the study, etc.), with one additional state being unresponsive, resulting in a final state count of 34 and a final sample size of 211,376 .

Following the methodology of Merrick and colleagues [11], states that included ACE items in their optional modules were contacted to establish data use agreements. The ACEs data from each state were merged (along with demographic and weighting variables) from 2009 to 2017 (see Table 1 and Fig. 2). Survey weights, which were included in the acquired data, were used to adjust the data to conform to population parameters provided by the CDC.

\section{Analytic strategy}

First, frequency statistics were computed for the overall sample, males, and females. Estimated weighted frequencies were further stratified by age, race/ethnicity, annual household income, employment status, educational attainment, sexual orientation, and four geographical regions- classified by the U.S. Census Bureau [28]. Next, a frequency analysis was conducted by each of the eight ACE categories using mean ACE scores, stratified by the same sociodemographic variables previously mentioned. Both sets of frequency analyses are weighted with corresponding 95\% confidence intervals (CI). Data analyses were conducted using SPSS software, version 24 [29]. Lastly, post-estimation F-tests were used to detect ACEs differences in mean scores (number of ACE exposures) and also differences within types of ACE exposure. We use the method outlined by Cumming [30] in which the 
Table 1 Shows the study breakdown by state and year

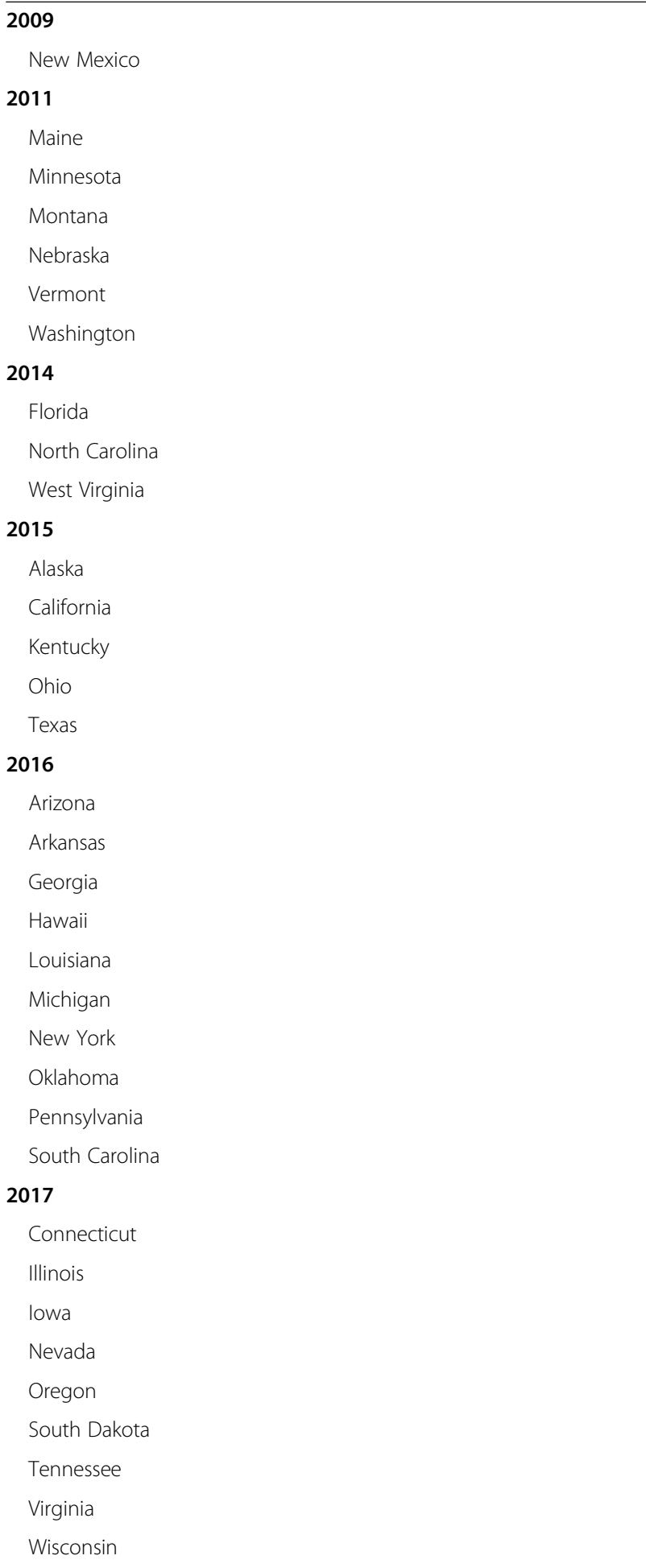

95\% confidence intervals of two coefficients are compared. In the event that the confidence intervals overlap by less than half the length of one confidence interval arm, then the $p$-value between the confidence intervals is at least below the level of significance (i.e., less than
.05). Previous studies show this method to be sufficiently accurate when two conditions are met: 1 . When sample sizes are at least 10 , and 2 . When the two intervals do not differ in width by more than a factor of 2 [30]. This applies to both dichotomous and continuous variables alike. All comparisons in this study met both assumptions. Further, our sample size is considerably large (in excess of 200,000). Thus, post-estimation F tests are more conservative than traditional group difference tests (e.g., $t$-tests and ANOVAs) and may protect against type 1 errors when analyze a larger sample [30]. It should be noted that this method has been used in other studies which utilized large samples [31, 32].

\section{Results}

\section{Population frequencies}

Table 2 presents the weighted estimates across demographic variables bifurcated by gender $(N=211,376)$. Generally, all age groups were represented, with the lowest percentage being 18 to 24 -year-olds (11.5\%) and the largest group being 64 and over (21.8\%). The majority of the sample was White $(65.1 \%)$ and identified as heterosexual/straight (95.8\%). Approximately one-third of the sample attended some college (31.3\%) with the next largest group having a high school degree (28.4\%), following by a college degree $(25.9 \%)$. The majority of individuals were employed (56.5\%) had income over $\$ 50$, 000 (47.6\%). Additionally, the majority of cases resided in the South (45.4\%) followed by the West (20.6\%), Midwest (20.1\%), and Northeast (13.7\%).

\section{Prevalence of ACEs}

In total, the majority of individuals experienced at least one adverse experience (57.8\%). Approximately $42 \%$ had an ACE score of 0 , followed by $22.9 \%$ (1 ACE), 12.8\% (2 ACEs), 8.2\% (3 ACEs), 5.7\% (4 ACEs), 3.8\% (5 ACEs), 2.3\% (6 ACEs), 1.2\% (7 ACEs), and 0.3\% (all 8 ACEs; not shown in tables). Table 3 presents the prevalence of ACEs by demographic variables among all eight ACE categories, as well as a total ACE mean score.

\section{ACE domains}

Overall, the most common type of ACE domain was emotional abuse (33.5\%), followed by parental separation/divorce (28.2\%), household substance abuse (26.8\%), IPV (17.8\%), physical abuse (17.5\%), household mental illness (16.2\%), sexual abuse (11.3\%), and incarcerated household member $(8.1 \%)$. The frequency of each ACE domain significantly differed from all other categories except for the prevalence between IPV and physical abuse. 


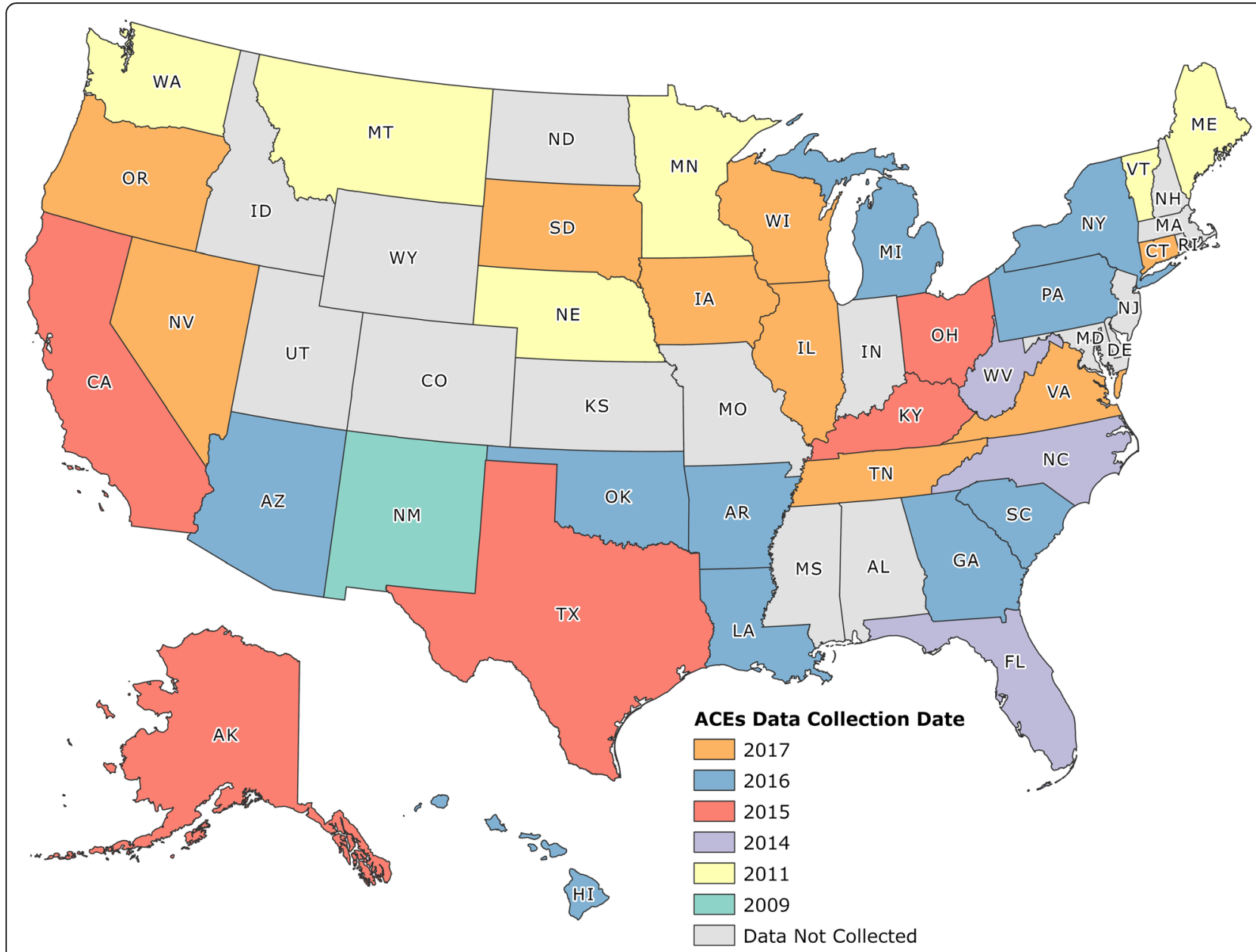

Fig. 2 Shows the data collected by state and year, graphically. Image was created by the author team

\section{Gender}

Post-estimation F-tests revealed that females had a significantly higher ACE score compared to males (1.64 to 1.46). Females had a significantly higher prevalence of adverse events in four of the eight categories (sexual, IPV, household substance abuse, and household mental illness), while males had a significantly higher prevalence of an incarcerated household member. No significant differences were found in emotional, physical, or divorce categories.

\section{Age}

F-tests showed that the 25 to 34 age group had a significantly higher ACE mean score than any other group (1.98), while the 64 and over group had a significantly lower ACE mean score than all other groups (0.94). With the exception of the 18 to 24 group compared to the 25 to 34 group, all groups differed significantly from one another. Of note, large disparities were found between the groups of 18 to 24 and 25 to 34 compared to all other older age groups in the categories of incarcerated household member and household mental illness.

\section{Race/ethnicity}

Individuals who identified as Multiracial had a significantly higher ACE mean score than all other races/ethnicities. This was also true for Multiracial individuals in six of the eight categories (emotional, physical, sexual, IPV, household substance abuse, and household mental illness). Individuals identifying as White had significantly lower mean ACE scores than those identifying as Black or Hispanic, while the "other" category had a significantly lower mean ACE score than all other categories.

\section{Household income}

Those making less than $\$ 15,000$ per year had a significantly higher mean ACE score compared to all other categories. This group also had a significantly higher prevalence than all other groups in each of the eight categories. The $\$ 15,000$ to $\$ 24,999$ group had significantly higher mean ACE scores than all higher 
Table 2 Demographic Characteristics of the Sample

\begin{tabular}{|c|c|c|c|c|c|c|c|c|c|}
\hline \multirow[b]{2}{*}{ Characteristic } & \multicolumn{3}{|l|}{ Male } & \multicolumn{3}{|l|}{ Female } & \multicolumn{3}{|l|}{ All } \\
\hline & No. & Wgt \% & $95 \% \mathrm{Cl}$ & No. & Wgt \% & $95 \% \mathrm{Cl}$ & No. & Wgt \% & $95 \% \mathrm{Cl}$ \\
\hline \multicolumn{10}{|l|}{ Age Group } \\
\hline $18-24$ & 4631 & 12.39 & (11.87-12.94) & 4268 & 10.74 & $(10.25-11.24)$ & 8900 & 11.53 & $(11.17-11.9)$ \\
\hline $25-34$ & 7469 & 15.61 & $(15.07-16.17)$ & 9321 & 14.43 & $(13.96-14.91)$ & 16,792 & 14.99 & $(14.64-15.36)$ \\
\hline $35-44$ & 9883 & 16.82 & $(16.28-17.37)$ & 13,019 & 15.47 & $(15.00-15.95)$ & 22,904 & 16.11 & $(15.75-16.48)$ \\
\hline $45-54$ & 14,706 & 18.09 & $(17.57-18.62)$ & 20,077 & 17.66 & $(17.2-18.14)$ & 34,791 & 17.87 & (17.52-18.22) \\
\hline $55-64$ & 20,365 & 17.31 & $(16.86-17.77)$ & 28,207 & 18.09 & $(17.66-18.53)$ & 48,575 & 17.72 & $(17.41-18.04)$ \\
\hline$>64$ & 28,751 & 19.78 & $(19.32-20.25)$ & 47,005 & 23.61 & $(23.16-24.06)$ & 75,761 & 21.78 & $(21.46-22.11)$ \\
\hline \multicolumn{10}{|l|}{ Race/Ethnicity } \\
\hline White & 67,131 & 65.17 & (64.44-65.88) & 95,550 & 64.98 & $(64.31-65.63)$ & 162,689 & 65.06 & (64.58-65.55) \\
\hline Black & 4838 & 10.66 & $(10.21-11.15)$ & 8689 & 11.93 & $(11.5-12.36)$ & 13,528 & 11.33 & $(11.01-11.65)$ \\
\hline Other & 4543 & 6.31 & $(5.92-6.72)$ & 5252 & 5.70 & $(5.31-6.13)$ & 9798 & 5.99 & $(5.71-6.29)$ \\
\hline Multiracial & 2303 & 1.45 & $(1.32-1.59)$ & 3009 & 1.39 & $(1.27-1.52)$ & 5314 & 1.42 & $(1.33-1.51)$ \\
\hline Hispanic & 5613 & 16.41 & $(15.8-17.04)$ & 7932 & 16.00 & $(15.45-16.57)$ & 13,546 & 16.20 & $(15.78-16.62)$ \\
\hline \multicolumn{10}{|l|}{ Household Income } \\
\hline$<15,000$ & 6179 & 8.98 & $(8.55-9.42)$ & 12,326 & 13.11 & $(12.64-13.59)$ & 18,505 & 11.08 & $(10.76-11.41)$ \\
\hline $15,000-24,999$ & 10,788 & 14.97 & $(14.43-15.52)$ & 18,947 & 19.03 & $(18.48-19.59)$ & 29,736 & 17.04 & $(16.65-17.43)$ \\
\hline $25,000-34,999$ & 8101 & 10.21 & $(9.78-10.67)$ & 12,130 & 10.84 & $(10.44-11.25)$ & 20,236 & 10.54 & $(10.24-10.84)$ \\
\hline $35,000-49,999$ & 11,432 & 14.22 & $(13.71-14.76)$ & 15,050 & 13.33 & $(12.89-13.79)$ & 26,486 & 13.77 & $(13.43-14.12)$ \\
\hline$>50,000$ & 39,473 & 51.62 & (50.89-52.36) & 42,919 & 43.69 & $(43.02-44.36)$ & 82,397 & 47.57 & $(47.07-48.07)$ \\
\hline \multicolumn{10}{|l|}{ Education } \\
\hline Less than HS & 6708 & 15.12 & $(14.52-15.73)$ & 9267 & 13.85 & $(13.34-14.36)$ & 15,975 & 14.45 & (14.06-14.85) \\
\hline HS diploma/GED & 24,332 & 29.51 & (28.89-30.14) & 34,282 & 27.32 & $(26.78-27.87)$ & 58,615 & 28.37 & $(27.96-28.78)$ \\
\hline Some college & 21,997 & 29.54 & $(28.91-30.18)$ & 35,299 & 32.89 & $(32.3-33.49)$ & 57,305 & 31.29 & $(30.86-31.73)$ \\
\hline College degree & 32,570 & 25.83 & (25.32-26.35) & 42,783 & 25.94 & $(25.46-26.43)$ & 75,362 & 25.89 & $(25.54-26.24)$ \\
\hline \multicolumn{10}{|l|}{ Employment Status } \\
\hline Employed & 47,133 & 64.70 & $(64.05-65.34)$ & 52,621 & 49.01 & $(48.38-49.63)$ & 99,766 & 56.50 & $(56.04-56.95)$ \\
\hline Unemployed & 4178 & 5.93 & (5.59-6.28) & 4805 & 5.29 & $(5-5.6)$ & 8984 & 5.59 & $(5.37-5.82)$ \\
\hline Unable to work & 5718 & 6.88 & $(6.54-7.24)$ & 10,016 & 8.41 & $(8.08-8.76)$ & 15,734 & 7.68 & $(7.44-7.93)$ \\
\hline Other & 27,798 & 22.49 & (21.96-23.03) & 53,206 & 37.29 & $(36.69-37.88)$ & 81,009 & 30.23 & $(29.82-30.64)$ \\
\hline \multicolumn{10}{|l|}{ Sexual Orientation } \\
\hline Straight & 44,748 & 96.14 & (95.81-96.44) & 60,072 & 95.48 & $(95.11-95.82)$ & 104,834 & 95.80 & (95.55-96.03) \\
\hline Gay/Lesbian & 935 & 2.08 & $(1.86-2.31)$ & 661 & 1.25 & $(1.09-1.46)$ & 1596 & 1.65 & $(1.52-1.81)$ \\
\hline Bisexual & 575 & 1.41 & $(1.23-1.63)$ & 1000 & 2.54 & $(2.28-2.82)$ & 1577 & 1.99 & $(1.83-2.17)$ \\
\hline Other & 167 & 0.37 & $(0.28-0.49)$ & 340 & 0.73 & $(0.59-0.89)$ & 507 & 0.56 & $(0.47-0.65)$ \\
\hline \multicolumn{10}{|l|}{ Census Region } \\
\hline Northeast & 14,396 & 13.70 & (13.26-14.14) & 19,530 & 13.77 & $(13.37-14.18)$ & 33,939 & 13.74 & $(13.44-14.04)$ \\
\hline South & 27,665 & 45.02 & $(44.34-45.71)$ & 42,265 & 45.78 & $(45.16-46.39)$ & 69,931 & 45.41 & $(44.96-45.87)$ \\
\hline Midwest & 21,205 & 20.26 & $(19.76-20.77)$ & 29,748 & 20.17 & $(19.72-20.63)$ & 50,958 & 20.22 & $(19.88-20.56)$ \\
\hline West & 22,539 & 21.02 & $(20.43-21.61)$ & 30,354 & 20.28 & $(19.73-20.84)$ & 52,895 & 20.63 & $(20.23-21.04)$ \\
\hline
\end{tabular}

earning groups, while the $\$ 50,000+$ category had significantly lower mean ACE scores compared to all groups.

\section{Educational attainment}

Individuals that earned a college degree had a significantly lower mean ACE score compared to all other groups. This was also true for ACE prevalence in six of the eight categories (physical, sexual, IPV, household substance abuse, divorce, and incarcerated household member). Those who earned less than a high school degree had a significantly higher prevalence of adversity in physical, IPV, and household substance abuse compared to all other categories. 
Table 3 Frequencies of ACE Types and Mean ACE Score by Sociodemographic Characteristics (Weighted Percent and 95\% Cl)

\begin{tabular}{|c|c|c|c|c|c|c|c|c|c|c|}
\hline & Emotional & Physical & Sexual & IPV & $\begin{array}{l}\text { House Sub. } \\
\text { Abuse }\end{array}$ & $\begin{array}{l}\text { Household } \\
\text { Mental III. }\end{array}$ & $\begin{array}{l}\text { Parental } \\
\text { Sep/Div. }\end{array}$ & $\begin{array}{l}\text { Incarcerated } \\
\text { Member }\end{array}$ & $\begin{array}{l}\text { ACE Score } \\
\text { Mean }\end{array}$ & $\begin{array}{l}\text { Mean Score } \\
\text { Sig. Diff. }\end{array}$ \\
\hline \multirow[t]{2}{*}{ Total } & 33.46 & 17.50 & 11.31 & 17.76 & 26.83 & 16.16 & 28.24 & 8.08 & 1.56 & \\
\hline & $\begin{array}{l}(33.02- \\
33.91)\end{array}$ & $\begin{array}{l}(17.14- \\
17.87)\end{array}$ & $\begin{array}{l}(11.02- \\
11.60)\end{array}$ & $\begin{array}{l}(17.40- \\
18.13)\end{array}$ & $\begin{array}{l}(26.42- \\
27.23)\end{array}$ & $(15.82-16.50)$ & $\begin{array}{l}(27.81- \\
28.67)\end{array}$ & $(7.81-8.36)$ & $(1.54-1.57)$ & \\
\hline \multicolumn{11}{|l|}{ Sex } \\
\hline \multirow[t]{2}{*}{ 1. Male } & 33.57 & 17.46 & 6.18 & 17.00 & 25.58 & 13.59 & 27.84 & 8.47 & 1.46 & $2>1$ \\
\hline & $\begin{array}{l}(32.91- \\
34.25)\end{array}$ & $\begin{array}{l}(16.92- \\
18.02)\end{array}$ & $\begin{array}{l}(5.87- \\
6.51)\end{array}$ & $\begin{array}{l}(16.46- \\
17.56)\end{array}$ & $\begin{array}{l}(24.98- \\
26.19)\end{array}$ & $(13.11-14.08)$ & $\begin{array}{l}(27.20- \\
28.48)\end{array}$ & $(8.06-8.90)$ & $(1.44-1.49)$ & \\
\hline \multirow[t]{2}{*}{ 2. Female } & 33.37 & 17.54 & 16.05 & 18.46 & 27.97 & 18.50 & 28.60 & 7.73 & 1.64 & \\
\hline & $\begin{array}{l}(32.77- \\
33.97)\end{array}$ & $\begin{array}{l}(17.06- \\
18.04)\end{array}$ & $\begin{array}{l}(15.59- \\
16.51)\end{array}$ & $\begin{array}{l}(17.97- \\
18.95)\end{array}$ & $\begin{array}{l}(27.42- \\
28.52)\end{array}$ & $(18.03-18.99)$ & $\begin{array}{l}(28.02- \\
29.19)\end{array}$ & $(7.38-8.09)$ & $(1.62-1.67)$ & \\
\hline \multicolumn{11}{|l|}{ Age Group } \\
\hline \multirow[t]{2}{*}{ 1. $18-24$} & 42.02 & 18.32 & 9.67 & 17.26 & 28.29 & 24.43 & 39.33 & 16.30 & 1.91 & $2>$ all \\
\hline & $\begin{array}{l}(40.29- \\
43.76)\end{array}$ & $\begin{array}{l}(17.00- \\
19.72)\end{array}$ & $\begin{array}{l}(8.71- \\
10.73)\end{array}$ & $\begin{array}{l}(16.01- \\
18.57)\end{array}$ & $\begin{array}{l}(26.80- \\
29.82)\end{array}$ & $(23.02-25.91)$ & $\begin{array}{l}(37.64- \\
41.05)\end{array}$ & $(15.07-17.61)$ & $(1.84-1.97)$ & $1>3,4,5,6$ \\
\hline \multirow[t]{2}{*}{ 2. $25-34$} & 40.05 & 21.07 & 11.42 & 21.71 & 31.52 & 23.05 & 40.72 & 14.10 & 1.98 & $3>4,5,6$ \\
\hline & $\begin{array}{l}(38.73- \\
41.39)\end{array}$ & $\begin{array}{l}(19.95- \\
22.24)\end{array}$ & $\begin{array}{l}(10.61- \\
12.27)\end{array}$ & $\begin{array}{l}(20.59- \\
22.88)\end{array}$ & $\begin{array}{l}(30.30- \\
32.78)\end{array}$ & $(21.96-24.18)$ & $\begin{array}{l}(39.39- \\
42.06)\end{array}$ & $(13.17-15.07)$ & $(1.93-2.04)$ & $4>5,6$ \\
\hline \multirow[t]{2}{*}{ 3. $35-44$} & 36.65 & 20.31 & 13.56 & 20.99 & 29.67 & 18.21 & 35.04 & 9.26 & 1.79 & $5>6$ \\
\hline & $\begin{array}{l}(35.46- \\
37.85)\end{array}$ & $\begin{array}{l}(19.31- \\
21.35)\end{array}$ & $\begin{array}{l}(12.75- \\
14.42)\end{array}$ & $\begin{array}{l}(19.98- \\
22.05)\end{array}$ & $\begin{array}{l}(28.57- \\
30.79)\end{array}$ & $(17.32-19.15)$ & $\begin{array}{l}(33.86- \\
36.23)\end{array}$ & $(8.57-9.99)$ & $(1.74-1.84)$ & \\
\hline \multirow[t]{2}{*}{ 4. $45-54$} & 35.69 & 19.11 & 13.62 & 19.11 & 28.08 & 15.52 & 29.07 & 6.44 & 1.63 & \\
\hline & $\begin{array}{l}(34.65- \\
36.74)\end{array}$ & $\begin{array}{l}(18.26- \\
19.99)\end{array}$ & $\begin{array}{l}(12.92- \\
14.35)\end{array}$ & $\begin{array}{l}(18.27- \\
19.98)\end{array}$ & $\begin{array}{l}(27.14- \\
29.04)\end{array}$ & $(14.80-16.28)$ & $\begin{array}{l}(28.09- \\
30.07)\end{array}$ & $(5.92-7.01)$ & $(1.59-1.67)$ & \\
\hline \multirow[t]{2}{*}{ 5. 55-64 } & 32.75 & 17.03 & 12.07 & 18.28 & 27.61 & 13.92 & 20.50 & 5.05 & 1.44 & \\
\hline & $\begin{array}{l}(31.85- \\
33.66)\end{array}$ & $\begin{array}{l}(16.31- \\
17.79)\end{array}$ & $\begin{array}{l}(11.47- \\
12.69)\end{array}$ & $\begin{array}{l}(17.53- \\
19.05)\end{array}$ & $\begin{array}{l}(26.79- \\
28.45)\end{array}$ & $(13.30-14.56)$ & $\begin{array}{l}(19.73- \\
21.28)\end{array}$ & $(4.63-5.51)$ & $(1.41-1.47)$ & \\
\hline \multirow[t]{2}{*}{ 6. $>64$} & 20.80 & 11.63 & 7.91 & 11.41 & 19.08 & 7.88 & 14.82 & 2.58 & 0.94 & \\
\hline & $\begin{array}{l}(20.18- \\
21.43)\end{array}$ & $\begin{array}{l}(11.14- \\
12.14)\end{array}$ & $\begin{array}{l}(7.54- \\
8.31)\end{array}$ & $\begin{array}{l}(10.92- \\
11.92)\end{array}$ & $\begin{array}{l}(18.49- \\
19.68)\end{array}$ & $(7.51-8.26)$ & $\begin{array}{l}(14.26- \\
15.39)\end{array}$ & $(2.37-2.82)$ & $(0.92-0.96)$ & \\
\hline \multicolumn{11}{|l|}{ Race/Ethnicity } \\
\hline \multirow[t]{2}{*}{ 1. White } & 34.01 & 16.35 & 11.23 & 15.95 & 27.62 & 18.18 & 26.00 & 6.67 & 1.53 & $4>$ all \\
\hline & $\begin{array}{l}(33.52- \\
34.15)\end{array}$ & $\begin{array}{l}(15.97- \\
16.74)\end{array}$ & $\begin{array}{l}(10.91- \\
11.55)\end{array}$ & $\begin{array}{l}(15.57- \\
16.34)\end{array}$ & $\begin{array}{l}(27.17- \\
28.08)\end{array}$ & $(17.78-18.59)$ & $\begin{array}{l}(25.53- \\
26.48)\end{array}$ & $(6.39-6.95)$ & $(1.51-1.54)$ & $2>1,3$ \\
\hline \multirow[t]{2}{*}{ 2. Black } & 29.97 & 13.46 & 12.30 & 20.88 & 25.87 & 12.22 & 43.84 & 14.45 & 1.66 & $5>1,3$ \\
\hline & $\begin{array}{l}(28.56- \\
31.41)\end{array}$ & $\begin{array}{l}(12.42- \\
14.57)\end{array}$ & $\begin{array}{l}(11.34- \\
13.33)\end{array}$ & $\begin{array}{l}(19.65- \\
22.16)\end{array}$ & $\begin{array}{l}(24.57- \\
27.21)\end{array}$ & (11.19-13.33) & $\begin{array}{l}(42.29- \\
45.41)\end{array}$ & $(13.37-15.60)$ & $(1.60-1.71)$ & $1>3$ \\
\hline \multirow[t]{2}{*}{ 3. Other } & 29.60 & 18.02 & 8.01 & 16.51 & 15.30 & 11.10 & 17.89 & 5.62 & 1.18 & \\
\hline & $\begin{array}{l}(27.35- \\
31.95)\end{array}$ & $\begin{array}{l}(16.19- \\
20.02)\end{array}$ & $\begin{array}{l}(6.89- \\
9.30)\end{array}$ & $\begin{array}{l}(14.81- \\
18.36)\end{array}$ & $\begin{array}{l}(13.73- \\
17.03)\end{array}$ & $(9.78-12.56)$ & $\begin{array}{l}(16.15- \\
19.77)\end{array}$ & $(4.68-6.75)$ & $(1.10-1.25)$ & \\
\hline \multirow[t]{2}{*}{ 4. Multiracial } & 47.12 & 27.10 & 19.29 & 27.26 & 39.31 & 26.78 & 44.60 & 14.95 & 2.39 & \\
\hline & $\begin{array}{l}(43.90- \\
50.37)\end{array}$ & $\begin{array}{l}(24.35- \\
30.04)\end{array}$ & $\begin{array}{l}(16.88- \\
21.96)\end{array}$ & $\begin{array}{l}(24.52- \\
30.19)\end{array}$ & $\begin{array}{l}(36.25- \\
42.45)\end{array}$ & $(24.04-29.71)$ & $\begin{array}{l}(41.41- \\
47.83)\end{array}$ & $(12.77-17.42)$ & $(2.26-2.51)$ & \\
\hline \multirow[t]{2}{*}{ 5. Hispanic } & 33.84 & 23.70 & 11.34 & 22.40 & 27.07 & 11.55 & 29.30 & 9.35 & 1.63 & \\
\hline & $\begin{array}{l}(32.43- \\
35.28)\end{array}$ & $\begin{array}{l}(22.45- \\
24.99)\end{array}$ & $\begin{array}{l}(10.46- \\
12.28)\end{array}$ & $\begin{array}{l}(21.19- \\
23.67)\end{array}$ & $\begin{array}{l}(25.80- \\
28.38)\end{array}$ & $(10.64-12.51)$ & $\begin{array}{l}(27.98- \\
30.67)\end{array}$ & $(8.51-10.27)$ & $(1.58-1.69)$ & \\
\hline \multicolumn{11}{|c|}{ Household Income } \\
\hline \multirow[t]{2}{*}{ 1. $<15,000$} & 37.70 & 25.16 & 16.31 & 25.06 & 33.89 & 19.33 & 36.73 & 12.64 & 2.00 & $1>$ all \\
\hline & $\begin{array}{l}(36.17- \\
39.25)\end{array}$ & $\begin{array}{l}(23.80- \\
26.57)\end{array}$ & $\begin{array}{l}(15.21- \\
17.47)\end{array}$ & $\begin{array}{l}(23.70- \\
26.46)\end{array}$ & $\begin{array}{l}(32.44- \\
35.38)\end{array}$ & $(18.13-20.60)$ & $\begin{array}{l}(35.19- \\
38.30)\end{array}$ & $(11.61-13.74)$ & $(1.97-2.06)$ & $2>3,4,5$ \\
\hline
\end{tabular}


Table 3 Frequencies of ACE Types and Mean ACE Score by Sociodemographic Characteristics (Weighted Percent and 95\% Cl) (Continued)

\begin{tabular}{|c|c|c|c|c|c|c|c|c|c|c|}
\hline & Emotional & Physical & Sexual & IPV & $\begin{array}{l}\text { House Sub. } \\
\text { Abuse }\end{array}$ & $\begin{array}{l}\text { Household } \\
\text { Mental III. }\end{array}$ & $\begin{array}{l}\text { Parental } \\
\text { Sep/Div. }\end{array}$ & $\begin{array}{l}\text { Incarcerated } \\
\text { Member }\end{array}$ & $\begin{array}{l}\text { ACE Score } \\
\text { Mean }\end{array}$ & $\begin{array}{l}\text { Mean Score } \\
\text { Sig. Diff. }\end{array}$ \\
\hline \multirow{2}{*}{$\begin{array}{l}\text { 2. } 15,000-24, \\
999\end{array}$} & 33.95 & 20.17 & 13.61 & 22.11 & 29.26 & 16.32 & 32.05 & 10.92 & 1.73 & $3>5$ \\
\hline & $\begin{array}{l}(32.73- \\
35.19)\end{array}$ & $\begin{array}{l}(19.13- \\
21.25)\end{array}$ & $\begin{array}{l}(12.77- \\
14.49)\end{array}$ & $\begin{array}{l}(21.00- \\
23.26)\end{array}$ & $\begin{array}{l}(28.13- \\
30.42)\end{array}$ & $(15.41-17.28)$ & $\begin{array}{l}(30.83- \\
33.29)\end{array}$ & $(10.08-11.82)$ & $(1.68-1.78)$ & $4>5$ \\
\hline \multirow{2}{*}{$\begin{array}{l}\text { 3. } 25,000-34 \\
999\end{array}$} & 33.21 & 18.79 & 12.00 & 19.49 & 27.84 & 15.17 & 28.23 & 8.85 & 1.59 & \\
\hline & $\begin{array}{l}(31.75- \\
34.70)\end{array}$ & $\begin{array}{l}(17.57- \\
20.08)\end{array}$ & $\begin{array}{l}(11.07- \\
12.99)\end{array}$ & $\begin{array}{l}(18.23- \\
20.80)\end{array}$ & $\begin{array}{l}(26.50- \\
29.22)\end{array}$ & $(14.10-16.31)$ & $\begin{array}{l}(26.85- \\
29.65)\end{array}$ & $(7.99-9.79)$ & $(1.54-1.65)$ & \\
\hline \multirow{2}{*}{$\begin{array}{l}\text { 4. } 35,000-49 \text {, } \\
999\end{array}$} & 35.03 & 18.08 & 11.83 & 18.90 & 27.45 & 15.83 & 29.49 & 8.88 & 1.62 & \\
\hline & $\begin{array}{l}(33.71- \\
36.37)\end{array}$ & $\begin{array}{l}(17.00- \\
19.21)\end{array}$ & $\begin{array}{l}(10.98- \\
12.73)\end{array}$ & $\begin{array}{l}(17.82- \\
20.02)\end{array}$ & $\begin{array}{l}(26.28- \\
28.66)\end{array}$ & $(14.87-16.83)$ & $\begin{array}{l}(28.22- \\
30.80)\end{array}$ & $(8.07-9.77)$ & $(1.56-1.67)$ & \\
\hline \multirow[t]{2}{*}{ 5. $>50,000$} & 33.76 & 15.48 & 9.83 & 15.27 & 25.24 & 16.80 & 25.13 & 5.77 & 1.44 & \\
\hline & $\begin{array}{l}(33.09- \\
34.44)\end{array}$ & $\begin{array}{l}(14.96- \\
16.02)\end{array}$ & $\begin{array}{l}(9.43- \\
10.25)\end{array}$ & $\begin{array}{l}(14.76- \\
15.79)\end{array}$ & $\begin{array}{l}(24.64- \\
25.85)\end{array}$ & $(16.27-17.34)$ & $\begin{array}{l}(24.50- \\
25.77)\end{array}$ & $(5.42-6.13)$ & $(1.42-1.47)$ & \\
\hline \multicolumn{11}{|c|}{ Educational Attainment } \\
\hline \multirow{2}{*}{$\begin{array}{l}\text { 1. Less than } \\
\mathrm{HS}\end{array}$} & 31.83 & 22.87 & 12.22 & 23.49 & 30.92 & 13.01 & 31.55 & 10.90 & 1.71 & $1>2,4$ \\
\hline & $\begin{array}{l}(30.40- \\
33.30)\end{array}$ & $\begin{array}{l}(21.59- \\
24.21)\end{array}$ & $\begin{array}{l}(11.26- \\
13.24)\end{array}$ & $\begin{array}{l}(22.20- \\
24.83)\end{array}$ & $\begin{array}{l}(29.55- \\
32.32)\end{array}$ & $(12.02-14.06)$ & $\begin{array}{l}(30.13- \\
33.00)\end{array}$ & $(9.99-11.89)$ & $(1.66-1.77)$ & $3>2,4$ \\
\hline \multirow{2}{*}{$\begin{array}{l}\text { 2. HS } \\
\text { diploma/ } \\
\text { GED }\end{array}$} & 32.12 & 17.37 & 10.43 & 18.21 & 27.55 & 14.73 & 31.17 & 9.94 & 1.57 & $2>4$ \\
\hline & $\begin{array}{l}(31.30- \\
32.96)\end{array}$ & $\begin{array}{l}(16.69- \\
18.06)\end{array}$ & $\begin{array}{l}(9.94- \\
10.95)\end{array}$ & $\begin{array}{l}(17.53- \\
18.91)\end{array}$ & $\begin{array}{l}(26.79- \\
28.32)\end{array}$ & $(14.10-15.38)$ & $\begin{array}{l}(30.35- \\
32.00)\end{array}$ & $(9.37-10.54)$ & $(1.54-1.61)$ & \\
\hline \multirow{2}{*}{$\begin{array}{l}\text { 3. Some } \\
\text { college }\end{array}$} & 36.88 & 18.42 & 13.38 & 18.74 & 29.08 & 18.85 & 30.80 & 8.56 & 1.70 & \\
\hline & $\begin{array}{l}(36.04- \\
37.72)\end{array}$ & $\begin{array}{l}(17.75- \\
19.10)\end{array}$ & $\begin{array}{l}(12.82- \\
13.96)\end{array}$ & $\begin{array}{l}(18.08- \\
19.43)\end{array}$ & $\begin{array}{l}(28.32- \\
29.84)\end{array}$ & (18.19-19.53) & $\begin{array}{l}(29.99- \\
31.63)\end{array}$ & (8.08-9.06) & $(1.67-1.73)$ & \\
\hline \multirow{2}{*}{$\begin{array}{l}\text { 4. College } \\
\text { degree }\end{array}$} & 31.82 & 13.61 & 9.36 & 13.03 & 21.11 & 16.25 & 20.24 & 3.95 & 1.26 & \\
\hline & $\begin{array}{l}(31.14- \\
32.50)\end{array}$ & $\begin{array}{l}(13.12- \\
14.11)\end{array}$ & $\begin{array}{l}(8.97- \\
9.76)\end{array}$ & $\begin{array}{l}(12.55- \\
13.52)\end{array}$ & $\begin{array}{l}(20.56- \\
21.68)\end{array}$ & $(15.74-16.77)$ & $\begin{array}{l}(19.67- \\
20.83)\end{array}$ & $(3.68-4.23)$ & $(1.24-1.29)$ & \\
\hline \multicolumn{11}{|c|}{ Employment Status } \\
\hline \multirow[t]{2}{*}{ 1. Employed } & 35.64 & 17.41 & 10.81 & 18.22 & 27.39 & 17.02 & 30.51 & 8.55 & 1.61 & $3>$ all \\
\hline & $\begin{array}{l}(35.02- \\
36.26)\end{array}$ & $\begin{array}{l}(16.91- \\
17.92)\end{array}$ & $\begin{array}{l}(10.43- \\
11.21)\end{array}$ & $\begin{array}{l}(17.71- \\
18.74)\end{array}$ & $\begin{array}{l}(26.83- \\
27.96)\end{array}$ & $(16.55-17.50)$ & $\begin{array}{l}(29.91- \\
31.11)\end{array}$ & $(8.17-8.93)$ & $(1.59-1.64)$ & \\
\hline \multirow{2}{*}{$\begin{array}{l}\text { 2. } \\
\text { Unemployed }\end{array}$} & 39.42 & 24.80 & 15.39 & 23.04 & 34.10 & 20.77 & 39.99 & 14.39 & 2.05 & $2>1,4$ \\
\hline & $\begin{array}{l}(37.39- \\
41.50)\end{array}$ & $\begin{array}{l}(22.97- \\
26.72)\end{array}$ & $\begin{array}{l}(13.99- \\
16.89)\end{array}$ & $\begin{array}{l}(21.33- \\
24.84)\end{array}$ & $\begin{array}{l}(32.17- \\
36.09)\end{array}$ & $(19.16-22.47)$ & $\begin{array}{l}(37.91- \\
42.11)\end{array}$ & $(12.84-16.09)$ & $(1.97-2.14)$ & $1>4$ \\
\hline \multirow{2}{*}{$\begin{array}{l}\text { 3. Unable to } \\
\text { work }\end{array}$} & 41.20 & 27.09 & 22.19 & 27.74 & 40.07 & 23.73 & 36.43 & 13.22 & 2.24 & \\
\hline & $\begin{array}{l}(39.55- \\
42.87)\end{array}$ & $\begin{array}{l}(25.61- \\
28.63)\end{array}$ & $\begin{array}{l}(20.77- \\
23.67)\end{array}$ & $\begin{array}{l}(26.24- \\
29.30)\end{array}$ & $\begin{array}{l}(38.46- \\
17.71)\end{array}$ & $(22.38-25.13)$ & $\begin{array}{l}(34.79- \\
38.09)\end{array}$ & $(12.08-14.46)$ & $(2.17-2.31)$ & \\
\hline \multirow[t]{2}{*}{ 4. Other } & 27.08 & 14.06 & 9.16 & 13.80 & 21.52 & 12.37 & 20.43 & 5.17 & 1.20 & \\
\hline & $\begin{array}{l}(26.34- \\
27.83)\end{array}$ & $\begin{array}{l}(13.49- \\
14.65)\end{array}$ & $\begin{array}{l}(8.74- \\
9.61)\end{array}$ & $\begin{array}{l}(13.25- \\
14.38)\end{array}$ & $\begin{array}{l}(20.89- \\
22.17)\end{array}$ & $(11.83-12.93)$ & $\begin{array}{l}(19.75- \\
21.12)\end{array}$ & $(4.79-5.59)$ & $(1.18-1.23)$ & \\
\hline \multicolumn{11}{|c|}{ Sexual Orientation } \\
\hline \multirow[t]{2}{*}{ 1. Straight } & 33.76 & 17.59 & 10.54 & 17.34 & 26.20 & 15.42 & 27.89 & 7.64 & 1.53 & \\
\hline & $\begin{array}{l}(33.19- \\
34.33)\end{array}$ & $\begin{array}{l}(17.13- \\
18.06)\end{array}$ & $\begin{array}{l}(10.20- \\
10.89)\end{array}$ & $\begin{array}{l}(16.88- \\
17.80)\end{array}$ & $\begin{array}{l}(25.70- \\
26.72)\end{array}$ & $(15.00-15.85)$ & $\begin{array}{l}(27.35- \\
28.44)\end{array}$ & $(7.31-7.98)$ & $(1.51-1.55)$ & \\
\hline \multirow{2}{*}{$\begin{array}{l}\text { 2. Gay/ } \\
\text { Lesbian }\end{array}$} & 48.05 & 28.77 & 23.60 & 27.68 & 36.73 & 26.31 & 33.41 & 12.13 & 2.30 & $3>$ all \\
\hline & $\begin{array}{l}(43.53- \\
52.60)\end{array}$ & $\begin{array}{l}(24.85- \\
33.05)\end{array}$ & $\begin{array}{l}(19.90- \\
27.75)\end{array}$ & $\begin{array}{l}(24.00- \\
31.69)\end{array}$ & $\begin{array}{l}(32.54- \\
41.13)\end{array}$ & $(22.78-30.18)$ & $\begin{array}{l}(29.41- \\
37.67)\end{array}$ & $(9.43-15.47)$ & $(2.11-2.49)$ & $2>1,4$ \\
\hline \multirow[t]{2}{*}{ 3. Bisexual } & 58.32 & 35.03 & 30.97 & 27.60 & 46.62 & 44.05 & 43.17 & 21.49 & 3.01 & $1>4$ \\
\hline & $\begin{array}{l}(53.95- \\
62.56)\end{array}$ & $\begin{array}{l}(30.96- \\
39.33)\end{array}$ & $\begin{array}{l}(27.18- \\
35.03)\end{array}$ & $\begin{array}{l}(24.01- \\
31.51)\end{array}$ & $\begin{array}{l}(42.37- \\
50.93)\end{array}$ & (39.79-48.39) & $\begin{array}{l}(38.88- \\
47.57)\end{array}$ & $(18.13-25.28)$ & $(2.83-3.20)$ & \\
\hline
\end{tabular}


Table 3 Frequencies of ACE Types and Mean ACE Score by Sociodemographic Characteristics (Weighted Percent and 95\% Cl) (Continued)

\begin{tabular}{|c|c|c|c|c|c|c|c|c|c|c|}
\hline & Emotional & Physical & Sexual & IPV & $\begin{array}{l}\text { House Sub. } \\
\text { Abuse }\end{array}$ & $\begin{array}{l}\text { Household } \\
\text { Mental III. }\end{array}$ & $\begin{array}{l}\text { Parental } \\
\text { Sep/Div. }\end{array}$ & $\begin{array}{l}\text { Incarcerated } \\
\text { Member }\end{array}$ & $\begin{array}{l}\text { ACE Score } \\
\text { Mean }\end{array}$ & $\begin{array}{l}\text { Mean Score } \\
\text { Sig. Diff. }\end{array}$ \\
\hline \multirow[t]{2}{*}{ 4. Other } & 34.75 & 21.73 & 9.68 & 17.45 & 24.66 & 13.72 & 25.47 & 6.58 & 1.50 & \\
\hline & $\begin{array}{l}(27.61- \\
42.64)\end{array}$ & $\begin{array}{l}(16.16- \\
28.58)\end{array}$ & $\begin{array}{l}(6.72- \\
13.74)\end{array}$ & $\begin{array}{l}(12.39- \\
24.01)\end{array}$ & $\begin{array}{l}(19.01- \\
31.33)\end{array}$ & $(9.89-18.71)$ & $\begin{array}{l}(19.33- \\
32.77)\end{array}$ & $(3.67-11.53)$ & $(1.22-1.79)$ & \\
\hline \multicolumn{11}{|l|}{ Census Region } \\
\hline \multirow[t]{2}{*}{ 1. Midwest } & 35.96 & 16.95 & 11.71 & 16.68 & 26.53 & 17.08 & 25.12 & 7.92 & 1.56 & $4>$ all \\
\hline & $\begin{array}{l}(35.07- \\
36.86)\end{array}$ & $\begin{array}{l}(16.24- \\
17.68)\end{array}$ & $\begin{array}{l}(11.12- \\
12.32)\end{array}$ & $\begin{array}{l}(15.99- \\
17.40)\end{array}$ & $\begin{array}{l}(25.71- \\
17.36)\end{array}$ & $(16.36-17.82)$ & $\begin{array}{l}(24.28- \\
25.98)\end{array}$ & $(7.36-8.51)$ & $(1.52-1.59)$ & \\
\hline \multirow[t]{2}{*}{ 2. Northeast } & 34.72 & 17.08 & 10.54 & 15.89 & 26.00 & 17.40 & 25.24 & 6.92 & 1.52 & \\
\hline & $\begin{array}{l}(33.61- \\
35.84)\end{array}$ & $\begin{array}{l}(16.22- \\
17.97)\end{array}$ & $\begin{array}{l}(9.85- \\
11.26)\end{array}$ & $\begin{array}{l}(15.04- \\
16.78)\end{array}$ & $\begin{array}{l}(25.00- \\
27.03)\end{array}$ & $(16.51-18.34)$ & $\begin{array}{l}(24.20- \\
26.31)\end{array}$ & $(6.31-7.58)$ & $(1.47-1.56)$ & \\
\hline \multirow[t]{2}{*}{ 3. South } & 29.85 & 15.96 & 11.42 & 17.83 & 25.99 & 15.60 & 29.55 & 8.23 & 1.49 & \\
\hline & $\begin{array}{l}(29.21- \\
30.51)\end{array}$ & $\begin{array}{l}(15.42- \\
16.51)\end{array}$ & $\begin{array}{l}(10.98- \\
11.87)\end{array}$ & $\begin{array}{l}(17.27- \\
18.40)\end{array}$ & $\begin{array}{l}(25.39- \\
26.59)\end{array}$ & $(15.10-16.12)$ & $\begin{array}{l}(28.89- \\
30.22)\end{array}$ & $(7.82-8.66)$ & $(1.47-1.52)$ & \\
\hline \multirow[t]{2}{*}{ 4. West } & 38.52 & 21.71 & 11.22 & 19.94 & 29.52 & 15.64 & 30.41 & 8.70 & 1.70 & \\
\hline & $\begin{array}{l}(39.36- \\
39.70)\end{array}$ & $\begin{array}{l}(20.79- \\
22.66)\end{array}$ & $\begin{array}{l}(10.57- \\
11.91)\end{array}$ & $\begin{array}{l}(19.05- \\
20.85)\end{array}$ & $\begin{array}{l}(28.52- \\
30.53)\end{array}$ & $(14.89-16.41)$ & $\begin{array}{l}(29.39- \\
31.45)\end{array}$ & (8.08-9.36) & $(1.66-1.74)$ & \\
\hline
\end{tabular}

Note: IPV Interpersonal Violence

\section{Employment status}

Those in the unable to work category had a significantly higher mean ACE score than all other employment categories (as well as six of the eight individual ACE categories), while those in the other category (including retirees, students, and homemakers) had a significantly lower mean ACE score than all other employment categories (also true in each of the eight individual ACE categories). It should be noted that the unemployed category had a significantly higher mean ACE score than those who were employed.

\section{Sexual orientation}

Bisexual individuals had a significantly higher prevalence of adversity in seven of eight categories (the exception being IPV) as well as high mean ACE scores. Of particular note, approximately $58 \%$ of bisexual individuals reported adversity in the emotional abuse category, the single highest percent of any adversity category across all groups in Table 3. Gay and lesbian individuals had significantly higher mean ACE scores than straight or "other" individuals.

\section{Census region}

Those residing in the West had a significantly higher mean ACE score compared to the other three regions (as well as four of the eight adversity categories including emotional, physical, IPV, and household substance abuse).

\section{Discussion}

The current study, to our knowledge, is the most diverse and comprehensive compilation of ACEs data and provides an expanded investigation of ACEs exposure across 34 states. Similar to Merrick and colleagues [11], our findings reveal ACEs are prevalent across all demographic variables. There are, however, some populations that experience higher rates of adversity compared to others. In particular, four categories showed particular vulnerabilities to ACEs: females, younger adults, sexual minorities, and multiracial individuals.

While the confidence intervals for females overlapped with the confidence intervals in males in seven of the eight categories, there was a substantial difference (and no confidence interval overlap) between the frequencies of sexual assault for females compared to males (16 to $6 \%$, respectively). Though this difference in stark, it seems unsurprising given past research has shown that while one in five women experience sexual assault, only one out of 70 men experience sexual assault [33], thus accounting for an overall higher mean ACE score in females. Generally, those who were younger reported higher mean ACEs than older individuals. Three possible rationales exist for these disparities. The first is that research has suggested that ACEs may be increasing [34]. Next, it is possible that individuals with higher ACEs may experience early death (thus these individuals are not representative in the data), as empirical evidence claims strong linkages between ACEs and shortened lifespans [35]. Lastly, it is possible that older individuals tend to minimize and/or fail to recall adverse childhood events [22], though this is less extensively studied in the 
literature. Additionally, there is currently a greater emphasis on/recognition of mental health issues when compared to past decades, thus creating the possibility that older individuals may not have perceived past events as markers of certain ACEs, such as familial mental illness.

With respect to individuals who identify as a sexual minority, our findings are similar to other studies which found a higher prevalence of adverse events among gay and bisexual individuals [36]. Though theories about why sexual minorities have higher ACEs have been postulated, such as certain types of abuse may catalyze shifts in sexual orientation or that sexual minorities may be more likely to recognize, and thus report, adverse events [36], the association of higher ACEs and sexual minorities remains unclear. Lastly, individuals who identified as multiracial had higher frequencies of ACEs than other races/ethnicities, though it should be noted that the frequencies in certain categories mirrored the frequencies of Black and/or Hispanic individuals. This aligns with other research highlighting that social and structural factors elevate the risk of childhood adversity, and that identifying as a racial/ethnic minority creates unique family stress that catalyzes adverse events [11,37].

Consistent with past research on ACEs, there were notable differences in mean ACE scores in the socioeconomic categories of education, income, and employment [11]. Income generally had a linear relationship with ACEs (i.e., greater income was associated with lower mean ACE scores), with the exception that making \$35, 000 to $\$ 49,999$ had a higher mean ACE score than those making $\$ 25,000$ to $\$ 34,000$, though the difference was not statistically significant.

With regard to education, there was no significant difference between less than high school and some college, while having a high school degree were associated with significantly lower ACEs. Having a college degree was significantly associated with the lowest ACEs. Data from the 2016 census revealed that more individuals have college degrees now more than ever, [38] and as such, it may be that the first three categories of education (all below having a college degree) represent a lower level of attainment compared to past decades where differences in these categories were more delineated- thus possibly explaining why there was no significant difference in having some college and not completing high school. It is also possible that some individuals were not old enough in order to complete a college degree, and thus, the data may be slightly skewed with respect to educational attainment; although it should be noted that the 18 to 24 age group only comprised $4 \%$ of the total sample.

With respect to employment status, those who were unemployed or out of work had high mean ACE scores than those who were employed. Because ACEs are associated with higher rates of disease and injury, it is possible that those with higher ACEs were unable to work due to a physical or mental ailment which impaired their ability to seek employment.

There is also a strong argument to be made regarding the impact that individual ACE domains have. Our results showed that emotional abuse was the most prevalent (33\%), while sexual abuse was the least prevalent (11\%), however, research has shown that these domains do not have equitable effects [39]. As such, the prevalence of ACEs domains should not be confused with correlations of impact (i.e., emotional abuse treated as a bigger issue than sexual abuse solely because of increased prevalence), particularly as programming is developed to limit childhood adversity and long-term sequelae.

\section{Conclusions}

Our study should be considered in conjunction with several limiting factors. As with all cross-sectional studies, causal inferences should not be taken as sacrosanct, as longitudinal data on adversity exposure is necessary. Next, although previous studies have established acceptable validity of self-reported adversity in childhood [40], the BRFSS relies on data that is self-reported, and thus, may be susceptible to memory and response biases [41]. Additionally, adversity is a complex, multi-dimensional set of processes that the ACEs framework attempts to simplify. There is an argument to be made that not all ACE categories are equal [42], and that protective processes may be just as important as adverse conditions across the lifespan [43]. The BRFSS does not account for multiple instances of a single adversity category (e.g., multiple instances of sexual abuse may be cumulatively as detrimental as experiencing adversity in multiple categories). It should also be noted that that the traditional ACE measure used in this study may not accurately reflect adversity experiences (particularly for individuals identifying as a racial/ethnic minority), and as such, calls for a more nuanced expansion of ACEs have been made [44].

Despite these limitations, our study has several implications for population-based public health. In particular, our study comprises the most comprehensive published ACEs dataset, which captures disparities across a broader geographic spectrum. This would be particularly helpful in a targeted campaign for specific demographic groups to help prevent ACEs. Nevertheless, while the prevention of ACEs is a complicated and difficult public health initiative, there is evidence to suggest that resilience and intervention programming for children aged 6 to 17 can help attenuate the deleterious effect of ACEs among children already experiencing adversity, [45] while protective factors (e.g., an adult who made a child 
feel safe and protected) have been shown to mitigate the effects of ACEs [46]. These programs would benefit from understanding ACEs from a population-based perspective, thus tailoring programs to those in high risk categories.

\section{Abbreviations}

ACEs: Adverse Childhood Experiences; IPV: Interpersonal Violence: BRFSS: Behavioral Risk Factor Surveillance System

\section{Acknowledgements}

We would like to acknowledge the state health departments which provided data: NM, ME, MN, MT, NE, VT, WA, FL, NC, WV, AK, CA, KY, OH, TX, AZ, AR, $G A, H I, L A, M I, N Y, O K, P A, S C, C T, I L, I A, N V, O R, S D, T N, V A$, and WI.

\section{Authors' contributions}

Authors ZG and RH secured data use agreements with each state and wrote the introduction, methods, and discussion. Author's ZG and DW conducted the analyses, constructed the tables, and wrote the results. The authors read and approved the final manuscript.

\section{Funding}

No funding to report.

\section{Availability of data and materials}

Original data was obtained from the Centers for Disease Control and Prevention's (CDC) Behavioral Risk Factor Surveillance System (BRFSS). Data are not available due to data sharing agreements with individual states.

\section{Ethics approval and consent to participate}

Data used in this publication were acquired with administrative permission from the following states: NM, ME, MN, MT, NE, VT, WA, FL, NC, WV, AK, CA, $K Y, O H, T X, A Z, A R, G A, H I, L A, M I I N Y, O K, P A, S C, C T, I L, I A, N V, O R, S D, T N$, $V A$, and WI.

\section{Consent for publication}

N/A

\section{Competing interests}

The authors declare that they have no competing interests.

Received: 22 April 2020 Accepted: 19 August 2020

Published online: 10 September 2020

\section{References}

1. Felitti $V$, Anda R, Nordenberg D, Williamson D. Adverse childhood experiences and health outcomes in adults: the ace study. J Fam Consum Sci. 1998;90(3):31.

2. Dong M, Dube SR, Felitti VJ, Giles WH, Anda RF. Adverse childhood experiences and self-reported liver disease: new insights into the causal pathway. Arch Intern Med. 2003;163(16):1949-56.

3. Danese A, Moffitt TE, Harrington H, Milne BJ, Polanczyk G, Pariante CM, et al. Adverse childhood experiences and adult risk factors for age-related disease: depression, inflammation, and clustering of metabolic risk markers. Arch Pediatr Adolesc Med. 2009;163(12):1135-43.

4. Brown DW, Anda RF, Felitti VJ, Edwards VJ, Malarcher AM, Croft JB, et al. Adverse childhood experiences are associated with the risk of lung cancer: a prospective cohort study. BMC Public Health. 2010;10(1):20.

5. Anda RF, Brown DW, Dube SR, Bremner JD, Felitti VJ, Giles WH. Adverse childhood experiences and chronic obstructive pulmonary disease in adults. Am J Prev Med. 2008;34(5):396-403.

6. Dong M, Giles WH, Felitti VJ, Dube SR, Williams JE, Chapman DP, et al. Insights into causal pathways for ischemic heart disease: adverse childhood experiences study. Circulation. 2004;110(13):1761-6.

7. Chapman DP, Whitfield CL, Felitti VJ, Dube SR, Edwards VJ, Anda RF. Adverse childhood experiences and the risk of depressive disorders in adulthood. J Affect Disord. 2004;82(2):217-25.

8. Sareen J, Henriksen C, Bolton S-L, TO A, Stein MB, Asmundson G. Adverse childhood experiences in relation to mood and anxiety disorders in a population-based sample of active military personnel. Psychol Med. 2013; 43(1):73-84.

9. Dube SR, Anda RF, Felitti VJ, Chapman DP, Williamson DF, Giles WH Childhood abuse, household dysfunction, and the risk of attempted suicide throughout the life span: findings from the adverse childhood experiences study. Jama. 2001:286(24):3089-96.

10. Lu W, Mueser KT, Rosenberg SD, Jankowski MK. Correlates of adverse childhood experiences among adults with severe mood disorders. Psychiatr Serv. 2008:59(9):1018-26.

11. Merrick MT, Ford DC, Ports KA, Guinn AS. Prevalence of adverse childhood experiences from the 2011-2014 behavioral risk factor surveillance system in 23 states. JAMA Pediatr. 2018;172(11):1038-44.

12. Roos LE, Mota N, Afifi TO, Katz LY, Distasio J, Sareen J. Relationship between adverse childhood experiences and homelessness and the impact of axis and II disorders. Am J Public Health. 2013:103(S2):S275-S81.

13. Pilowsky DJ, Keyes KM, Hasin DS. Adverse childhood events and lifetime alcohol dependence. Am J Public Health. 2009:99(2):258-63.

14. Dasgupta N, Beletsky L, Ciccarone D. Opioid crisis: no easy fix to its social and economic determinants. Am J Public Health. 2018:108(2):182-6.

15. Whetten K, Leserman J, Lowe K, Stangl D, Thielman N, Swartz M, et al. Prevalence of childhood sexual abuse and physical trauma in an HIV-positive sample from the deep south. Am J Public Health. 2006;96(6):1028-30.

16. Zierler S, Feingold L, Laufer D, Velentgas P, Kantrowitz-Gordon I, Mayer K. Adult survivors of childhood sexual abuse and subsequent risk of HIV infection. Am J Public Health. 1991;81(5):572-5.

17. Schilling EA, Aseltine RH, Gore S. Adverse childhood experiences and mental health in young adults: a longitudinal survey. BMC Public Health. 2007;7(1):30

18. Brody GH, Yu T, Chen E, Miller GE. Family-centered prevention ameliorates the association between adverse childhood experiences and prediabetes status in young black adults. Prev Med. 2017;100:117-22.

19. Verbitsky-Savitz N, Hargreaves MB, Penoyer S, Morales N, Coffee-Borden B, Whitesell E. Preventing and mitigating the effects of ACEs by building community capacity and resilience: APPI cross-site evaluation findings. Washington, DC: Mathematica Policy Research; 2016.

20. Centers for Disease Control and Prevention. The BRFSS data user quide. Atlanta: Department of Health and Human Services; 2013.

21. Sacks V, Murphey D. The prevalence of adverse childhood experiences, nationally, by state, and by race or ethnicity. Child Trends. 2018;20:2018.

22. Felitti VJ, Anda RF, Nordenberg D, Williamson DF, Spitz AM, Edwards V, et al. Relationship of childhood abuse and household dysfunction to many of the leading causes of death in adults: the adverse childhood experiences (ACE) study. Am J Prev Med. 2019;56(6):774-86.

23. Shaked D, Williams M, Evans MK, Zonderman AB. Indicators of subjective social status: differential associations across race and sex. SSM-Popul Health. 2016;2:700-7.

24. Halfon N, Larson K, Son J, Lu M, Bethell C. Income inequality and the differential effect of adverse childhood experiences in US children. Acad Pediatr. 2017:17(7):S70-S8

25. Lanier P, Maguire-Jack K, Lombardi B, Frey J, Rose RA. Adverse childhood experiences and child health outcomes: comparing cumulative risk and latent class approaches. Matern Child Health J. 2018;22(3):288-97.

26. Shonkoff JP. Capitalizing on advances in science to reduce the health consequences of early childhood adversity. JAMA Pediatr. 2016;170(10): $1003-7$

27. Ford DC, Merrick MT, Parks SE, Breiding MJ, Gilbert LK, Edwards VJ, et al. Examination of the factorial structure of adverse childhood experiences and recommendations for three subscale scores. Psychol Violence. 2014;4(4):432.

28. US Census Bureau. Census regions and divisions of the United States. US Census Bureau website. Retried from: https://www2.census.gov/geo/pdfs/ mapsdata/maps/reference/us_regdiv.pdf. 2010.

29. Cor I. IBM SPSS statistics for windows, version 24.0. Armonk: IBM Corp; 2016

30. Cumming G. Inference by eye: reading the overlap of independent confidence intervals. Stat Med. 2009;28(2):205-20.

31. Ene D, Der G, Fletcher-Watson S, O'Carroll S, MacKenzie G, Higgins M, et al. Associations of socioeconomic deprivation and preterm birth with speech, language, and communication concerns among children aged 27 to 30 months. JAMA Netw Open. 2019:2(9):e1911027.

32. Takayanagi Y, Spira AP, Roth KB, Gallo JJ, Eaton WW, Mojtabai R. Accuracy of reports of lifetime mental and physical disorders: results from the Baltimore epidemiological catchment area study. JAMA Psychiatry. 2014;71(3):273-80. 
33. Smith SG, Zhang X, Basile KC, Merrick MT, Wang J, Kresnow M-j, et al. The national intimate partner and sexual violence survey: 2015 data briefupdated release; 2018.

34. Vannorsdall TD, Munro CA. The link between childhood adversity and latelife mental health: evidence for the influence of early-life experiences or illusory correlations? Int Psychogeriatr. 2017;29(3):357-8.

35. Anda RF, Dong M, Brown DW, Felitti VJ, Giles WH, Perry GS, et al. The relationship of adverse childhood experiences to a history of premature death of family members. BMC Public Health. 2009;9(1):106.

36. Andersen JP, Blosnich J. Disparities in adverse childhood experiences among sexual minority and heterosexual adults: results from a multi-state probability-based sample. PLoS One. 2013;8(1):e54691.

37. Sedlak AJ, Mettenburg J, Basena M, Peta I, McPherson K, Greene A. Fourth national incidence study of child abuse and neglect (NIS-4), vol. 9. Washington, DC: US Department of Health and Human Services; 2010.

38. Wilson R. Census: more Americans have college degrees than ever before, vol. 5: The Hill; 2017

39. Negriff S. ACEs are not equal: examining the relative impact of household dysfunction versus childhood maltreatment on mental health in adolescence. Soc Sci Med. 2020;245:112696

40. Hardt J, Rutter M. Validity of adult retrospective reports of adverse childhood experiences: review of the evidence. J Child Psychol Psychiatry. 2004;45(2):260-73.

41. Tourangeau R, Yan T. Sensitive questions in surveys. Psychol Bull. 2007; 133(5):859.

42. Nurius PS, Logan-Greene P, Green S. ACEs within a social disadvantage framework: distinguishing unique, cumulative, and moderated contributions to adult mental health. J Prev Interv Community. 2012;40(4):278.

43. Morris AS, Treat A, Hays-Grudo J, Chesher T, Williamson AC, Mendez J. Integrating research and theory on early relationships to guide intervention and prevention. In: Building early social and emotional relationships with infants and toddlers: springer; 2018. p. 1-25.

44. Cronholm PF, Forke CM, Wade R, Bair-Merritt MH, Davis M, Harkins-Schwarz $M$, et al. Adverse childhood experiences: expanding the concept of adversity. Am J Prev Med. 2015:49(3):354-61.

45. Bethell CD, Newacheck P, Hawes E, Halfon N. Adverse childhood experiences: assessing the impact on health and school engagement and the mitigating role of resilience. Health Aff. 2014;33(12):2106-15.

46. Crouch E, Radcliff E, Strompolis M, Srivastav A. Safe, stable, and nurtured: protective factors against poor physical and mental health outcomes following exposure to adverse childhood experiences (ACEs). J Child Adolesc Trauma. 2019;12(2):165-73.

\section{Publisher's Note}

Springer Nature remains neutral with regard to jurisdictional claims in published maps and institutional affiliations.

Ready to submit your research? Choose BMC and benefit from:

- fast, convenient online submission

- thorough peer review by experienced researchers in your field

- rapid publication on acceptance

- support for research data, including large and complex data types

- gold Open Access which fosters wider collaboration and increased citations

- maximum visibility for your research: over $100 \mathrm{M}$ website views per year

At $\mathrm{BMC}$, research is always in progress.

Learn more biomedcentral.com/submissions 